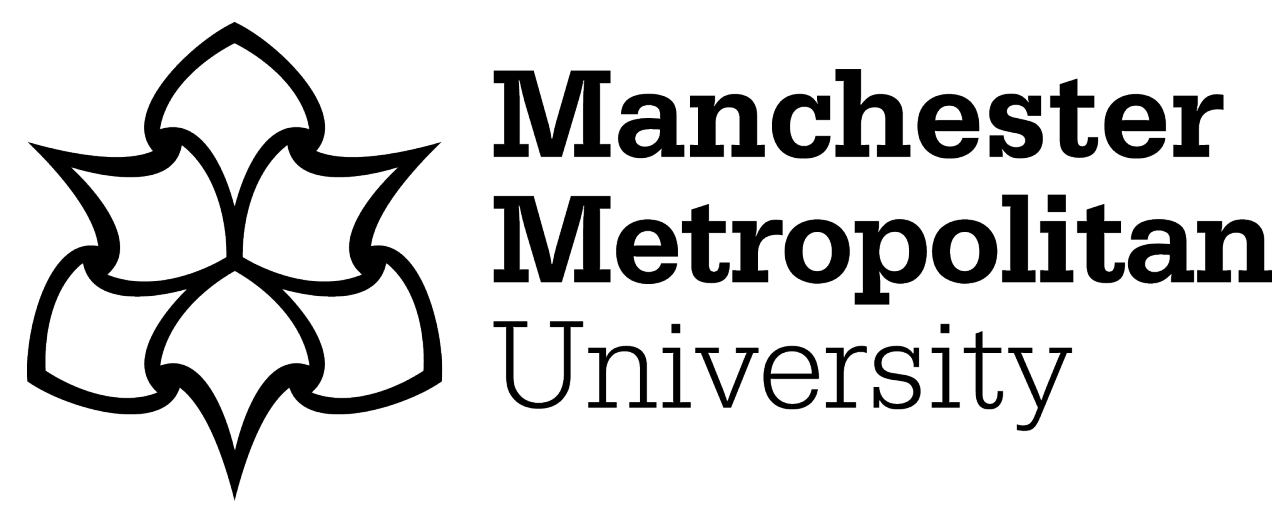

Denovan, AM ORCID logoORCID: https://orcid.org/0000-0002-9082-7225, Dagnall, N ORCID logoORCID: https://orcid.org/0000-0003-0657-7604, Dhingra, K and Grogan, S ORCID logoORCID: https://orcid.org/0000-00027510-765X (2017) Evaluating the Perceived Stress Scale among UK university students: implications for stress measurement and management. Studies in Higher Education, 44 (1). pp. 120-133. ISSN 0307-5079

Downloaded from: https://e-space.mmu.ac.uk/618671/

Version: Accepted Version

Publisher: Taylor \& Francis (Routledge)

DOI: https://doi.org/10.1080/03075079.2017.1340445

Please cite the published version 


\title{
Evaluating the Perceived Stress Scale among UK university students: implications for stress measurement and management
}

\author{
Andrew Denovan ${ }^{a^{*}}$, Neil Dagnall ${ }^{\mathrm{a}}$, Katie Dhingra ${ }^{\mathrm{b}}$ and Sarah Grogan ${ }^{\mathrm{a}}$ \\ ${ }^{a}$ Department of Psychology, Manchester Metropolitan University, Manchester, UK; \\ ${ }^{b}$ Department of Social Sciences, Leeds Beckett University, Leeds, UK \\ *Corresponding author: Andrew Denovan, Department of Psychology, Manchester \\ Metropolitan University, 53 Bonsall St, Manchester, M15 6GX, UK. E-mail: \\ a.denovan@mmu.ac.uk
}

\begin{abstract}
University life can be stressful, and accurate measurement of perceived stress is important for research and practice. However, despite widespread use, disagreement persists regarding the latent structure of the Perceived Stress Scale (PSS), which poses serious consequences for how the measure should be administered. Furthermore, factorial invariance between genders has not been established with the 10-item PSS, though gender differences in perceived stress have been detected. This study examined the factor structure, composite reliability, convergent validity, and gender invariance of the PSS-10 among 524 UK university students. Four distinct factor models (one-factor, correlated two-factor, correlated three-factor, and bifactor) were examined using confirmatory factor analysis. The totality of results supported a bifactor solution. Multi-group analysis established configural, metric, and scalar invariance of this model across gender. This study supports the use of total PSS-10 scores with UK university students and suggests the scale is not significantly affected by gender bias.
\end{abstract}

Keywords: confirmatory factor analysis; factorial invariance; Perceived Stress Scale; stress management; UK university students.

The final publication of this article is available at Taylor \& Francis via: http://dx.doi.org/10.1080/03075079.2017.1340445 


\section{Introduction}

The Perceived Stress Scale (PSS; Cohen, Kamarch, and Mermelstein 1983) is a global stress measure that assesses the extent to which respondents perceive life to be unpredictable, uncontrollable and overloading (Golden-Kreutz et al. 2004). The PSS contains general rather than event-specific items and is sensitive to background extraneous stressors and existing stressful circumstances. The original PSS contained 14-items. However, subsequent scrutiny produced a modified 10-item version (PSS-10), which possesses superior psychometric properties (internal consistency and factor structure) (Cohen and Williamson 1988). Consequently, Cohen and Williamson (1988) recommended that researchers use the PSS-10 rather than the original version.

Whilst the PSS-10 is a widely used, succinct measure of perceived stress, its factorial structure is a source of contention. Particularly, debate centres on whether the PSS-10 is uni or multi-dimensional. During initial development of the original measure, Cohen and Williamson (1988) identified an item distinction related to statement directionality (negative vs. positive). Acknowledging this, the authors concluded that the PSS-10 assessed a single construct, explaining $41.6 \%$ of data variance. Other studies, however, argue that the distinction represents separate factors (negative items assess Perceived Helplessness, and positive, Perceived Self-Efficacy) (Barbosa-Leiker et al. 2013; Golden-Kreutz et al. 2004; Roberti, Harrington, and Storch 2006).

Further work advances an alternative bifactor model (Jovanović and GavrilovJerković 2015; Wu and Amtmann 2013). Bifactor modelling assessed whether the PSS-10 was sufficiently unidimensional to warrant the use of total scores, and examined the additional contribution of orthogonal factors to specific scale items (Reise, Morizot, and Hays 2007). The PSS-10 bifactor solution comprises an underlying general perceived stress factor with two domain specific factors (Factor 1, negatively and Factor 2, positively worded items). 
Wu and Amtmann (2013) found this solution produced superior fit to previously advanced one and two-factor models.

Dissimilar solutions arise in part from the use of different statistical techniques and sample heterogeneity. For example, Cohen and Williamson's (1988) single factor interpretation emerged from exploratory factor analysis (orthogonal rotation) of data from a non-clinical U.S. sample $(N=2387)$. Confirmatory factor analysis $(\mathrm{CFA})$, which assesses data fit to a priori theoretical assumptions, advocates alternative factorial solutions. A further complication is the diverse range of samples included within psychometric validation studies. Illustratively, several student-based studies support the two-factor structure. Wongpakaran and Wongpakaran (2010) tested 479 Thai medical students, Örücü and Demir (2009) assessed 508 Turkish undergraduates, and Roberti et al. (2006) collected data from 285 American undergraduates. Contrastingly, support for the bifactor model originates from 446 participants with multiple sclerosis. Furthermore, Bradbury (2013), using a non-clinical Australian sample $(N=194)$, found superior fit for a correlated three-factor model (comprising Distress, Coping, and Emotional Reactivity factors).

Another important issue concerns the extent to which emergent factorial solutions apply similarly to men and women; an issue that has received limited attention despite reports that women score significantly higher (Gitchel, Roessler, and Turner 2011; Martin, Kazarian, and Breiter 1995). Indeed, most models assume gender invariance. Studies examining factor scores have found that women score significantly higher on Distress, but not Coping (Gitchel et al. 2011; Martin et al. 1995). A potential explanation is that gender differences arise from measurement bias rather than true differences between men and women. Bias in measurement occurs when one gender is more likely to endorse particular items, leading to artificial inflation of scores for specific items. Gitchel et al. (2011) investigated this issue using 1310 individuals with multiple sclerosis and discovered that women typically endorsed items on 
the Distress factor. Lavoie and Douglas (2012), however, using the PSS, found support for configural, metric, and scalar invariance of Distress and Coping factors across gender $(N=$ 1,636). Results can be criticised for reliance on correlating errors (see Brown 2006).

Smith, Rosenberg, and Haight (2014) found gender invariance for the PSS-10 among 557 U.S. undergraduates, but practiced factor cross-loading, which can pose problems for discriminant validity (Farrell 2010). Taylor (2015) examined gender invariance of the PSS-10 and obtained mixed results, invariance in relation to factor loadings and intercepts for a correlated two-factor model was not established. Clearly, factorial analysis of the PSS-10 needs to consider gender invariance to elucidate the source of gender differences.

Issues arising from analytical and conceptual inconsistencies (i.e., structural ambiguity, sample heterogeneity and gender invariance) potentially limit PSS-10 application, interpretation and usefulness. Particularly, the appropriateness of generally consulted scoring schemes to particular samples is questionable. Effective interpretation of PSS-10 data requires an appreciation of its psychometric properties within specific groups. Correspondingly, the present study examined PSS-10 factorial structural and gender invariance within a UK university sample. A similar approach generated normative Englishsample data for the Short Form Perceived Stress Scale (PSS-4) (Warttig et al. 2013). This focus was required for several reasons. Explicitly, to date few studies have examined the applicability of the PSS-10 to UK students. Hence, the measure is frequently used, but its effectiveness (with this group) is not fully evaluated. Indeed, researchers in other countries have recently conducted similar country specific analyses (e.g., Denmark, Nielsen et al. 2016; and Germany, Klein et al. 2016).

The present study used UK university students because they are a significant, frequently studied group, who encounter myriad stressors (i.e., university transition, combining employment with study, self-funding of education, academic demands) (Denovan 
and Macaskill 2016). Though university is largely a positive, challenging experience, evidence indicates that UK university students report higher levels of stress than general population averages (Turner et al. 2015). Research shows that the high stress levels among students are not restricted to the UK, with reported stress levels exceeding general population averages in Sweden (Vaez, Kristenson, and Laflamme 2004), Canada (Adlaf, Demers, and Gliksman 2005), the U.S. (Blanco et al. 2008), and Australia (Stallman, 2010). Key elements predicting stress are coping ability and the degree to which individuals view events as threatening (Lazarus and Folkman 1984).

Within the UK student group accurate measurement and conceptualisation of perceived stress is vital because high stress levels are associated with a range of negative outcomes, including depression (Zhang et al. 2015), suicidal ideation (Abdollahi et al. 2015), and lower subjective well-being (Denovan and Macaskill 2016). Problems appear particularly acute within students below the age of 26 because they are undertaking the transition to adulthood (Macaskill 2013). Thus, informed measurement of perceived stress is fundamental to facilitate understanding of susceptibility to and the nature of psychological problems. In addition, to use valid and reliable measures of stress is important for University Counselling services to gain an accurate understanding of the levels of stress affecting students seeking help, and to provide an indication of the appropriate mental health support that needs to be provided (Roberti et al. 2006).

The PSS-10 was studied as a measure of student stress rather than other established student-stress scales, such as the Inventory of College Students' Recent Life Experiences (ICSRLE; Kohn, Lafreniere, and Gurevich 1990), for various reasons. Specifically, the PSS10 is simple to administer, easy to complete, and scale brevity facilitates the inclusion of additional measures which might otherwise be excluded due to test battery length (Smith et al. 2014); a concern with measures including the ICSRLE (a 49-item scale). Alongside 
pragmatic expedience, the PSS-10 is versatile and can be used in a number of important educational contexts. For instance, to evaluate progress during counselling and to measure student distress. Conceptually, researchers and practitioners value the PSS-10 because items are non-specific and context free, and it can be used across a range of settings.

\section{The present study}

This study evaluated the psychometric properties of the PSS-10 with a UK university student sample. Comparison of factorial model fit indicated which solution was most applicable to the target sample. Measures of invariance investigated whether construct measurement varied as a function of gender (tests assessed configural, metric and scalar invariance). Additionally, composite reliability provided a robust examination of internal measurement integrity. Lastly, correlations between PSS-10 scores (total and subscale), and measures of student hassle exposure and affective well-being (positive and negative emotion) acted as validity measures. Given the perception of stress is related to both the frequency of hassle exposure (Kohn et al. 1990) and affective well-being (Schiffrin and Nelson 2010), it was hypothesised that PSS-10 scores would correlate positively with greater hassles and lower well-being.

\section{Method}

\section{Participants}

Five hundred and twenty-four social science university students (300 women, 57\% and 224 men, $43 \%$ ) were recruited via convenience sampling from a large post-92 UK university with a 2015/16 intake of 32,485 students. The university is classed as post-92 because it is one of the UK universities that were polytechnics until 1992. The courses students were studying included law, criminology, sociology, politics, psychology, and business. Participant mean age was $20.08, S D=3.70$; ages ranged from 18 to 42 . The majority $(90 \%)$ were in the age range of 18 to 23 , while the remainder (10\%) were in the age range of 24 to 42 . The mean age for women was $19.45, S D=2.80$; range of 18 to 42 , and the mean age for men was $20.94, S D$ 
$=4.51$; range of 18 to 41 . The difference between mean ages in relation to gender was significant, $t(522)=-4.67, p<.001$. Whilst at university, $204(39 \%)$ lived at home, 320 (61\%) lived in halls of residence or student houses/flats, and $236(45 \%)$ worked in paid employment.

\section{Measures}

The PSS-10 (Cohen and Williamson 1988) measures important components of stress by assessing how uncontrollable, overloaded, and unpredictable individuals find their lives. The PSS-10 asks about thoughts and feelings over the last month using a response scale from 0 (never) to 4 (very often). Cohen and Williamson (1988) reported good internal reliability ( $\alpha=$ .78) with two-day test-retest reliability of .85 . In the current study $\alpha=.86$ for the total scale, .84 for the Distress factor, and .76 for the Coping factor. An example Distress item is "how often have you felt nervous and 'stressed'?" (item 3), and an example Coping item is "how often have you felt that things were going your way?" (item 5).

The ICSRLE (Kohn et al. 1990) assesses university student stress as hassle exposure and contains seven subscales (developmental challenge, time pressure, academic alienation, romantic problems, assorted annoyances, general social mistreatment, friendship problems). Respondents hassle exposure frequency over the past month from 1 (not at all part of my life) to 4 (very much part of my life). It has good internal reliability $(\alpha=.89)$ (Kohn et al., 1990). In this study $\alpha=.88$ for the total scale, .76 for developmental challenge, .76 for time pressure, .78 for academic alienation, .67 for romantic problems, .66 for assorted annoyances, .78 for social mistreatment, and .72 for friendship problems.

The Positive and Negative Affectivity Schedule (PANAS) (Watson, Clark, and Tellegen 1988) measures basic and specific positive and negative emotions. The inventory contains two mood scales, 10 positive affect (PA) items (e.g., interested, proud) and 10 negative affect (NA) items (e.g., guilty, hostile). Participants rate items on a scale of 1 (very 
slightly) to 5 (extremely). To maintain consistency with the state measures of stress used in this study, the PANAS state version was administered, where participants rate the degree to which they experienced each emotion in the past month. Reported internal reliability is good $(\alpha=.90$ for PA, .87 for NA) as is test-retest reliability $(\mathrm{PA}=.61, \mathrm{NA}=.71)$ (Watson et al. 1988). In this study $\alpha=.88$ for PA, and .85 for NA.

\section{Procedure}

University students were invited to participate via introductory classes across the Social Sciences Department in week one of the first semester, and were provided with questionnaires to complete in situ. The beginning of the university year was focused on because this represents an important transitional point for all students. Students enrol and have induction, and they experience uncertainty about the year ahead in terms of timetable, workload and the nature of assessments (Struthers, Perry, and Menec 2000). Additionally, surveys/tests located at the beginning of the year are less likely to be influenced by proactive interference. In this context, previous stressors may cause rumination and worry, which produce residual stress. The beginning of the academic year is typically a point when student focus is forward (prospective) rather than retrospective and reactive (Roediger, Putnam, and Smith 2011). The University Research Ethics Committee approved the study. Participants were informed of the study's purpose, assured of confidentiality, and were debriefed after taking part.

\section{Analysis}

Four competing models of the latent factor structure of the PSS-10, based on previous research, were assessed using confirmatory factor analysis (CFA). Model 1 is a one-factor solution where the 10 items of the PSS-10 load onto a single latent variable of perceived stress. Model 2 is a correlated two-factor model where the two latent variables are represented by Distress (items 1, 2, 3, 6, 9, 10) and Coping (items 4, 5, 7, 8). Model 3 is a 
correlated three-factor model where the three latent variables are represented by Distress (items 6, 10, 2, 8), Coping (items 5, 4, 7), and Emotional Reactivity (items 1, 3, 9). Model 4 is a bifactor model consisting of three latent factors: a general perceived stress factor (PS Total), Distress and Coping. Items were constrained to load onto a single factor within models 1 to 3. Within model 4, all items loaded onto PS Total, items 1, 2, 3, 6, 9, 10 loaded onto Distress, and items 4, 5, 7, 8 loaded onto Coping. The labelling of factors as Distress, Coping, and Emotional Reactivity is consistent with previous research (e.g., Bradbury 2013). The superior factor solution was assessed for measurement invariance across gender using multi-group CFA.

A range of fit indices were used to evaluate the fit of each model. The chi-square $\left(\chi^{2}\right)$ statistic assesses both the covariance matrix and the sample, with good fitting models indicated by a non-significant result. However, chi-square is heavily influenced by sample size, and otherwise good fitting models are often rejected. Accordingly, other indices were considered. The Comparative Fit Index (CFI; Cronbach 1990) and the Tucker-Lewis Index (TLI; Tucker and Lewis 1973) evaluate the extent to which a model fits the data better than a baseline model where the variables are uncorrelated. Values above .95 suggest good fit, and values of .90 and greater suggest adequate fit (Hu and Bentler 1999). The Standardized RootMean-Square Residual (SRMR: Jöreskog and Sörbom 1981) and the Root-Mean-Square Error of Approximation (RMSEA: Steiger 1990) were also considered, with values less than .05 suggesting good fit, and values less than .08 suggesting reasonable fit (Hu and Bentler 1999). The $90 \%$ confidence interval (CI) was included when reporting RMSEA. Modification indices (MI) were examined to identify parameter misfit. This index reflects the degree to which the model chi-square would decrease if a constrained parameter was freely estimated. Within-item error correlations possessing MI > 20 were examined (ten Klooster et al. 2008). 
Akaike's Information Criterion (AIC; Akaike 1974) was used for model comparison, with smaller values signifying better fitting models.

Through multi-group CFA, three models were tested in relation to the superior factor solution: configural invariance, weak factorial invariance (metric invariance), and strong factorial invariance (scalar invariance). Byrne (2010) emphasises that testing for strict factorial invariance is unnecessary as this is rarely achievable. Testing for configural invariance focuses on the extent to which the same number of factors represents the data of both groups (Byrne 2010). In testing for metric invariance, all factor loadings were constrained to be equal between the two groups (Hair et al. 1998). Scalar invariance was tested by constraining the intercepts of the 10 items of the PSS-10 to be the same between the two groups. The CFI and chi-square differences between the models were considered. The CFI difference should not exceed .02 (Cheung and Rensvold, 1999), and chi-square should not significantly differ between models. Satisfaction of these criteria indicates that the PSS10 is not biased in relation to its factor structure, factor loadings, or intercepts (Milfont \& Fischer 2015).

\section{Results}

\section{Descriptive statistics}

The mean PSS-10 score for the sample was $19.79(S D=6.37)$. No gender difference in PSS10 total score was found, $t(522)=1.66, p=.09$, indicating that women $(M=20.19, S D=$ $6.03)$ reported similar levels of perceived stress to men $(M=19.25, S D=6.76)$. Participants reported slightly higher positive $\operatorname{mood}(M=33.14, S D=7.31)$ than negative $\operatorname{mood}(M=$ 24.68, $S D=7.84)$. The mean ICSRLE Total score was $75.03(S D=19.09)$. All skewness and kurtosis values were in the recommended interval between -2 to +2 (Byrne 2010) (see Table 1). 
[Table 1 here]

\section{Confirmatory factor analysis}

For the one-factor model of the PSS-10, the fit indices demonstrated unacceptable fit on all indices but SRMR: $\chi^{2}(35, N=524)=299.97, p<.001, \mathrm{CFI}=.85, \mathrm{TLI}=.81, \mathrm{SRMR}=.07$, RMSEA $=.12$ (CI of .11 to .13). Modification indices suggested the presence of high error covariances between items 1 and 2, 4 and 7,5 and 7,5 and 8, 7 and 8. Allowing these error terms to correlate significantly improved fit: $\chi^{2}$ difference $(5, N=524)=195.26, p<.001$. The fit indices indicated good model fit for the correlated two-factor model: $\chi^{2}(34, N=524)$ $=108.21, p<.001, \mathrm{CFI}=.96, \mathrm{TLI}=.95, \mathrm{SRMR}=.03, \mathrm{RMSEA}=.06(\mathrm{CI}$ of .05 to .08$) . \mathrm{MI}$ values reported high error covariance for items 1 and 2 . Allowing these error terms to correlate significantly improved fit: $\chi^{2}$ difference $(1, N=524)=42.26, p<.001$.

The correlated three-factor model suggested a reasonable fit to the data on all indices but RMSEA and TLI: $\chi^{2}(32, N=524)=200.50, p<.001, \mathrm{CFI}=.91, \mathrm{TLI}=.87, \mathrm{SRMR}=$ $.06, \mathrm{RMSEA}=.10$ (CI of .09 to .11). MI values reported high error covariance for items across factors (items 1 and 2, 7 and 8). These error terms were not allowed to correlate given cross-factor error correlations are discouraged (Byrne 2010). The bifactor model demonstrated superior fit in comparison with the one-factor, two-factor, and three-factor models: $\chi^{2}(25, N=524)=45.72, p=.007, \mathrm{CFI}=.99, \mathrm{TLI}=.98, \mathrm{SRMR}=.02, \mathrm{RMSEA}=.04$ (CI of .02 to .06). The AIC statistics further confirm the superior fit of the bifactor model, as the AIC is 125.72, which is lower than the one-factor (AIC =339.97), the one-factor with correlated errors $(\mathrm{AIC}=174.71)$, the two-factor $(\mathrm{AIC}=170.21)$, the two-factor with correlated errors $(\mathrm{AIC}=129.95)$, and the three-factor model $(\mathrm{AIC}=266.50)$.

[Table 2 here]

The suitability of the bifactor model can furthermore be determined by its parameter estimates. All factor loadings for PS Total were positive and statistically significant, and the 
majority exceeded .4, with the exception being items 7 and 4 (loadings of .39 and .37 respectively) (see Figure 1). Further scrutiny of the loadings for the two subscale factors offers critical information in relation to the appropriateness of incorporating these subscales when scoring the PSS-10. In situations when items load highly onto a general factor, but simultaneously load to a lesser degree onto subscale factors, this indicates that a unidimensional scoring method would be apposite (Reise, Moore, and Haviland 2010). However, when items load more highly onto subscale factors than a general factor, this supports the creation of subscales. In terms of the subscales, factor loadings for Distress were comparatively weaker than for PS Total, but Coping displayed robust factor loadings. Only items 1 and 2 significantly loaded onto Distress, and unexpectedly two items loaded negatively (items 6 and 10) which can be a result of the cross-over suppression effect (Paulhus et al. 2004). These results support the superiority of a single latent factor underlying the PSS-10, and to a lesser extent support the presence of two separate subscales.

[Figure 1 here]

\section{Multi-group analysis (gender)}

Factorial invariance tests were performed by fitting the superior model (the bifactor solution) to the data for men $(n=224)$ and women $(n=300)$. The bifactor model was used as a baseline for examining a hierarchy of progressively restrictive models (Bollen 1989), beginning with configural invariance, metric invariance, and scalar invariance. Results supported invariance of form (configural invariance) for the bifactor model, indicating good model fit: $\chi^{2}(52, N=524)=63.32, p=.14, \mathrm{CFI}=.99, \mathrm{TLI}=.99, \mathrm{SRMR}=.03, \mathrm{RMSEA}=$ $.02(90 \% \mathrm{CI}$ of .01 to .04$)$.

The test for the invariance of factor loadings (metric invariance) represented a good fit: $\chi^{2}(67, N=524)=75.28, p=.23, \mathrm{CFI}=.99, \mathrm{TLI}=.99, \mathrm{SRMR}=.03, \mathrm{RMSEA}=.02(90 \%$ CI of .01 to .03). Because the metric invariance model is nested within the baseline model, a 
$\chi^{2}$ difference test was performed, which revealed a non-significant result: $\chi^{2}(15, N=524)=$ $11.95, p=.68$. The difference between CFI values did not exceed .02 (configural CFI $=.99$, metric CFI $=.99$ ), supporting the conclusion of invariance at configural and metric stages.

The scalar invariance model reported a good fit to the data: $\chi^{2}(77, N=524)=84.97$, $p=.25, \mathrm{CFI}=.99, \mathrm{TLI}=.99, \mathrm{SRMR}=.03, \mathrm{RMSEA}=.02(\mathrm{CI}$ of .01 to .03$) . \mathrm{A} \chi^{2}$ significance test comparing the scalar model with the metric model supported the presence of strong factorial invariance: $\chi^{2}(10, N=524)=9.68, p=.46$. Comparison of CFI values reported a difference less than .02 , confirming strong factorial invariance (metric CFI = .99, scalar CFI = .99). These findings suggest that the PSS-10 is factorially invariant between men and women in relation to the bifactor solution.

\section{Reliability analysis}

Internal consistency tests including Cronbach's $\alpha$ often under- or overestimate scale reliability within a latent modelling context, and composite reliability offers a more rigorous assessment of internal consistency (Raykov 1998). The composite reliability of the bifactor scales were investigated with values greater than .60 considered acceptable (Diamantopoulos and Siguaw 2000). Results indicate that the PS Total factor and Coping factor possess satisfactory internal consistency ( $\rho c=.88$ and $\rho c=.70$ respectively). In contrast, composite reliability for the Distress factor was lower $(\rho c=.10)$.

\section{Convergent validity}

To assess convergent validity of the bifactor scales, correlations were computed between PS Total, Distress and Coping with PANAS subscales (positive affect, PA; negative affect, NA) and the ICSRLE (including subscales of developmental challenge, time pressure, academic alienation, romantic problems, assorted annoyances, general social mistreatment, and friendship problems) (see Table 3).

[Table 3 here] 
The correlations are in the expected direction for PA and NA, as PS Total, Distress, and Coping are significantly positively correlated with NA and significantly negatively correlated with PA. PS Total demonstrates a comparatively weaker albeit significant relationship with ICSRLE Total. PS Total and Distress were significantly associated with subscales of developmental challenge and time pressure, whereas Coping was significantly associated with friendship problems. Overall, the PSS-10 demonstrates convergent validity with the PANAS, and to a lesser extent the ICSRLE in this study.

\section{Discussion}

This study investigated the latent structure, factorial invariance, internal consistency and convergent validity of the PSS-10 among a large sample of UK university students. Participants had relatively high levels of perceived stress $(M=19.79, S D=6.37)$ compared with previously established norms with a general population sample aged 18-29 years $(M=$ 14.2, $S D=6.2$; Cohen 1994). Based on the fit indices from CFA, the bifactor model, consisting of PS Total, Distress and Coping was a closer fit to the data than one-factor, twofactor, and three-factor alternatives. Additionally, greater item loadings were evident for PS Total than Distress and, to an extent, Coping. Such findings suggest that although individual stress and coping factors exist, the PSS-10 is driven by a single underlying dimension of perceived stress. These results are consistent with Wu and Amtmann (2013) and indicate that total scores are usable when assessing UK university students as the PSS-10 is sufficiently unidmensional. This is a significant outcome and provides evidence for the unidimensionality assumption of the PSS-10, supporting Cohen and Williamson's (1988) original conclusion as to how the measure should be conceptualized. Furthermore, support for the unidimensionality assumption of the PSS-10 has recently been established with student samples using alternative psychometric methods. Specifically, Medvedev et al. (2017) used Rasch analysis and confirmed that the PSS-10 possesses an underlying latent factor of perceived stress. 
In relation to previous research with student samples (e.g., Örücü and Demir 2009; Roberti et al. 2006; Wongpakaran and Wongpakaran 2010), the correlated two-factor solution provided a good fit to the data, but none of the fit indices showed an improvement over those observed for the bifactor model. These results conflict with the assertion of Martin et al. (1995) that the PSS measures two facets of stress that are substantially distinct. Furthermore, in contrast with Bradbury (2013), a comparison of three-factor and two-factor models resulted in a superior fit for two-factor conceptualizations of perceived stress. An outcome that needs to be acknowledged, however, is the observation that items maintained relatively high factor loadings on Coping in the bifactor solution. It is unclear why this occurred, but Perera et al. (2016) recently found that the four positively stated items which make up Coping can act as a nuisance variable that introduce unwanted variation when scoring the PSS, which may explain the high loadings in this study. Perera et al. (2016) suggest that to obtain total scores and scores based on the positively stated items may help to control for this potential variance when administering the PSS.

From factorial invariance testing, configural, metric, and scalar invariance across gender were found in the present study for a bifactor solution, which is new in the literature. This suggests that PSS-10 scores may not be majorly influenced by a bias in how men and women treat the items. Concern regarding potential gender bias resulted from past research reporting that women had a higher probability of endorsing the Distress factor items relative to men (Gitchel et al. 2011). Yet, similar to this study, Lavoie and Douglas (2012) found no evidence of a gender bias. This disparity among studies may exist because different procedures of assessing measurement bias (e.g., item-response techniques) can produce contrasting results (Millsap 2006). The finding that the structure and factor loadings of the PSS-10 did not vary between men and women has important implications for interpreting mean differences in relation to gender. 
Contrary to expectations and the results of Gitchel et al. (2011), although women reported a higher mean difference in perceived stress, this was not significant. It is not clear why this occurred, but the outcome of invariance across gender adds to a growing body of research indicating that observed differences in mean stress among men and women are unlikely to be artefacts of measurement bias, but instead represent true mean differences arising from psychological, biological, or social factors. Additionally, results support the use of the PSS-10 for assessing gender differences in stress among university students, given the absence of gender bias in this study.

Determining the most suitable latent structure of a measure is an important precondition to evaluating its reliability, as to treat a unidimensional scale as multidimensional can lead to inaccurate reliability assessment (Shevlin et al. 2000). Accordingly, following testing for internal consistency using Cronbach's alpha, the composite reliability of the factors of the best fitting model were assessed to robustly evaluate internal consistency. Composite reliability was satisfactory for PS Total and Coping, but not for Distress. These findings reinforce the notion of a general PS factor in the conceptualization of the PSS-10.

Further analysis supported the validity of the scale for assessing university student stress, as PS Total, Distress and Coping significantly correlated with affective well-being. PS Total significantly correlated with total university-based hassles, and PS Total and Distress were associated with developmental challenge and time pressure. Coping, however, only correlated with friendship problems. It is not immediately apparent why low correlations between the PSS-10 and ICSRLE emerged. A possible reason is that the ICSRLE contains study-related stress items specific to a North American population, and perhaps these items did not transfer adequately to a UK student sample. Indeed, Bodenhorn et al. (2007) report that research using the ICSRLE has been largely confined to North American students, and 
UK-based studies using the ICSRLE tend to use adapted versions of the measure, which was not practiced in this study. Future research may want to consider stress measures that are not confounded by such issues when assessing PSS-10 convergent validity with UK students, such as the Stress subscale of the Depression Anxiety Stress Scale (Lovibond \& Lovibond 1995).

\section{Implications and limitations}

This study indicates that the PSS-10 provides a psychometrically sound and, in comparison with multi-scale measures of stress (e.g., the ICSRLE), a brief and easy to administer unidimensional stress measure for university students. The scale is efficacious not only for research studies that require a number of variables, but also for practical settings. The PSS-10 is not designed to assess psychological symptomatology, but is useful for suggesting who may be at risk for clinical disorders (Cohen and Williamson 1988). The measure can therefore be usefully applied within university settings as a screening tool to judge those in need of further support, who can then be linked to University Counselling services. In addition, for University Counselling services to administer a measure at the beginning of treatment which can detect high levels of stress can provide important insight in terms of a student's life situations and their ability to cope. Lowered stress scores throughout treatment can indicate a positive treatment response, and the measure can be used as a parsimonious means of assessing how students are negotiating difficult situations in response to stress management techniques (Roberti et al. 2006).

Findings should be interpreted in light of several limitations. First, the student sample was relatively homogeneous, limiting the generalizability of the results to samples of varying ages and backgrounds. Replication with more heterogeneous samples is needed. Particularly, the age range was quite restrictive, with only $10 \%$ of the sample belonging to the age category of 24-42. Although the age range is consistent with related research (e.g., Smith et 
al. 2014), future work would benefit from considering more mature students to further evaluate the PSS-10. Additionally, the focus on social science students does not represent the range of subjects at the entire university. Given much research focusing on student stress utilises social science (and specifically psychology) students, this may not represent a serious limitation. Second, the use of self-report data introduces several well-known limitations, such as response bias and shared method variance. Future research would benefit from including additional measures when assessing perceived stress, such as interviews and physiological assessment. Finally, this study did not assess test-retest reliability of the PSS-10. Previous research has, however, established temporal reliability in a variety of populations (see Reis, Hino, and Añez 2010).

\section{Conclusion}

Despite the limitations, this study contributes to research literature surrounding the PSS in several ways. Firstly, support for a bifactor model in a large sample of UK university students indicates that, in agreement with Wu and Amtmann (2013), the PSS is best conceptualized as being driven by a single underlying dimension of perceived stress, suggesting that total PSS10 scores are appropriate to use for assessing UK university student stress. Convergent validity of the PSS-10 implies that this scale accurately surveyed perceived stress among the student sample. Results also indicate that the underlying constructs are interpreted and measured the same way across gender, with configural, metric and scalar invariance demonstrated between men and women. This finding adds to the general literature in terms of how mean differences between genders in perceived stress should be interpreted. The scale overall provides a parsimonious means of assessing stress among UK university students for research and practical settings. Additionally, the present study has provided the first indications of factorial invariance of a bifactor solution across gender, and presented robust evidence of the reliability of such a conceptualization. Considering the findings in 
conjunction with those of Wu and Amtmann (2013), Taylor (2015), and Lavoie and Douglas (2012) from other samples provides strong evidence for the appropriateness of a bifactor model and gender invariance in relation to the PSS-10. 


\section{References}

Abdollahi, A., M.A. Talib, S.N. Yaacob, and Z. Ismail. 2015. "The role of hardiness in decreasing stress and suicidal ideation in a sample of undergraduate students." Journal of Humanistic Psychology 55(2): 202-222. doi:10.1177/0022167814543952

Adlaf, E.M., A. Demers, and L. Gliksman. 2005. Canadian campus survey 2004. Toronto, ON: Centre for Addiction and Mental Health.

Akaike, H. 1974. "A new look at the statistical model identification." IEEE Transactions on Automatic Control 19(6): 716-23. doi:10.1109/TAC.1974.1100705

Barbosa-Leiker, C., M. Kostick, S. McPherson, V. Roper, T. Hoekstra, and B. Wright. 2013. "Measurement invariance of the perceived stress scale and latent mean differences across gender and time.” Stress and Health 21(2): 253-260. doi:10.1002/smi.2463

Blanco, C., M. Okuda, C. Wright, D.S. Hasin, B.F. Grant, S. Liu, and M. Wolfson. (2008). "Mental health of college students and their non-college attending peers." Archives of General Psychiatry 65(12): 1429-1437. doi:10.1001/archpsyc.65.12.1429

Bodenhorn, N., Y. Miyazaki, K. Ng, and C. Zalaquett. 2007. "Analysis of the Inventory of College Students' Recent Life Experiences." Multicultural Learning and Teaching 2(2): 65-77.

Bollen, K.A. 1989. Structural equations with latent variables. New York, NY: Wiley.

Bradbury, J. 2013. "Modelling stress constructs with biomarkers: The importance of the measurement model." Clinical and Experimental Medical Sciences 1(5-8): 197-216. doi:10.12988/cems.2013.13017

Brown, T.A. 2006. Confirmatory factor analysis for applied research. New York, NY: Guilford Press.

Byrne, B.M. 2010. Structural equation modeling with Amos: Basic concepts, applications, and programming. 2nd ed. New York, NY: Taylor and Francis. 
Cheung, G.W., and R.B. Rensvold 2002. "Evaluating goodness-of-fit indexes for testing measurement invariance." Structural Equation Modeling 9(2): 233-255. doi:10.1207/S15328007SEM0902_5

Cohen, S. 1994. Perceived Stress Scale. Palo Alto, CA: Mind Garden.

Cohen, S., T. Kamarch, and R. Mermelstein. 1983. "A global measure of perceived stress." Journal of Health and Social Behavior 24(4): 385-396. doi:10.2307/2136404

Cohen, S., and G. Williamson 1988. "Perceived stress in a probability sample of the U.S." In The social psychology of health, edited by S. Spacapan and S. Oskamp, 31-67. Thousand Oaks, CA: Sage.

Cronbach, L.J. 1990. Essentials of psychological testing. 5th ed. New York, NY: Harper and Row.

Denovan, A., and A. Macaskill. 2016. "Stress and subjective well-being among first year UK undergraduate students.” Journal of Happiness Studies. Advance online publication. doi:10.1007/s10902-016-9736-y

Diamantopoulos, A., and J.A. Siguaw. 2000. Introducing LISREL. London, UK: Sage.

Farrell, A.M. 2010. "Insufficient discriminant validity: A comment on Bove, Pervan, Beatty, and Shiu 2009.” Journal of Business Research 63(3): 324-327. doi:10.1016/j.jbusres.2009.05.003

Gitchel, W.D., R.T. Roessler, and R.C. Turner 2011. “Gender effect according to item directionality on the perceived stress scale for adults with multiple sclerosis." Rehabilitation Counselling Bulletin 55(1): 20-28. doi:10.1177/0034355211404567

Golden-Kreutz, D.M., M.W. Browne, G.M. Frierson, and B.L. Anderson. 2004. “Assessing stress in cancer patients: A second-order factor analysis model for the Perceived Stress Scale.”Assessment 11(3): 216-222. doi:10.1177/1073191104267398 
Hair, J.F., R.E. Anderson, R.L. Tatham, and W.C. Black. 1998. Multivariate data analysis. 5th ed. Upper Saddle River, NJ: Prentice Hall.

Hu, L., and P.M. Bentler. 1999. "Cutoff criteria for fit indexes in covariance structure analysis: Conventional criteria versus new alternatives." Structural Equation Modeling 6(1): 1-55. doi:10.1080/10705519909540118

Jöreskog, K., and D. Sörbom. 1981. LISREL V: Analysis of linear structural relationships by the method of maximum likelihood. Chicago, IL: National Educational Resources.

Jovanović, V., and V. Gavrilov-Jerković. 2015. "More than a (negative) feeling: Validity of the Perceived Stress Scale in Serbian clinical and non-clinical samples.” Psihologija 48(1): 5-18. doi:10.2298/PSI1501005J

Klein, E.M., E. Brähler, M. Dreier, L. Reinecke, K.W. Müller, G. Schmutzer, K. Wölfling, and M.E. Beutel. 2016. "The German version of the Perceived Stress Scale: psychometric characteristics in a representative German community sample.” BMC psychiatry 16: 159. doi:10.1186/s12888-016-0875-9

Kohn, P.M., K. Lafreniere, and M. Gurevich. 1990. “The Inventory of College Student's Recent Life Experiences: A decontaminated hassles scale for a special population." Journal of Behavioural Medicine 13(6): 619-630. doi:10.1007/BF00844738

Lavoie, J.A.A., and K.S. Douglas. 2012. "The Perceived Stress Scale: Evaluating configural, metric and scalar invariance across mental health status and gender." Journal of Psychopathology and Behavioral Assessment 34(1): 48-57. doi:10.1007/s10862-0119266-1

Lazarus, R.S., and S. Folkman. 1984. Stress, appraisal, and coping. New York, NY: Springer.

Lovibond, P.F., and S.H. Lovibond. "The structure of negative emotional states: Comparison of the Depression Anxiety Stress Scales (DASS) with the Beck Depression and 
Anxiety Inventories." Behaviour research and therapy 33(3): 335-343. doi:10.1016/0005-7967(94)00075-U

Macaskill, A. 2013. "The mental health of university students in the United Kingdom." British Journal of Guidance \& Counselling 41(4): 426-441. doi:10.1080/03069885.2012.743110

Martin, R.A., S.S. Kazarian, and H.J. Breiter. 1995. "Perceived stress, life events, dysfunctional attitudes, and depression in adolescent psychiatric inpatients." Journal of Psychopathology \& Behavioral Assessment 17(1): 81-95. doi:10.1007/BF02229205

Medvedev, O. N., C. U. Krägeloh, E.M. Hill, R. Billington, R.J. Siegert, C.S. Webster, R.J. Booth, and M.A. Henning. 2017. "Rasch analysis of the Perceived Stress Scale: Transformation from an ordinal to a linear measure." Journal of Health Psychology. Advance online publication. doi:10.1177/1359105316689603

Milfont, T. L., and R. Fischer. 2015. “Testing measurement invariance across groups: Applications in cross-cultural research.” International Journal of Psychological Research 3(1): 111-121. doi:10.21500/20112084.857

Millsap, R.E. 2006. "Comments on methods for the investigation of measurement bias in the Mini-Mental State Examination.” Medical Care 44(11): 171-175. doi:10.1097/01.mlr.0000245441.76388.ff

Nielsen, M.G., E. Ørnbøl, M. Vestergaard, P. Bech, F.B. Larsen, M. Lasgaard, and K.S. Christensen. 2016. "The construct validity of the Perceived Stress Scale.” Journal of psychosomatic research 84: 22-30. doi:10.1016/j.jpsychores.2016.03.009

Örücü, Ç.M., and A. Demir. 2009. "Psychometric evaluation of Perceived Stress Scale for Turkish university students." Stress and Health 25(1): 103-109. doi:10.1002/smi.1218

Paulhus, D.L., R.W. Robins, K.H. Trzesniewski, and J.L. Tracy. 2004. “Two replicable suppressor situations in personality research.” Multivariate Behavioral Research 39(2): 303-328. doi:10.1207/s15327906mbr3902_7 
Perera, M.J., C.E. Brintz, O. Birnbaum-Weitzman, F.J. Penedo, L.C. Gallo, P. Gonzalez, N. Gouskova et al. 2016. "Factor structure of the Perceived Stress Scale-10 (PSS) across English and Spanish language responders in the HCHS/SOL Sociocultural Ancillary Study." Psychological Assessment. Advance online publication. doi:10.1037/pas0000336

Raykov, T. 1998. "Coefficient alpha and composite reliability with interrelated nonhomogeneous items.” Applied Psychological Measurement 22(4): 375-385. doi:10.1177/014662169802200407

Reis, R.S., A.A. Hino, and C.R. Añez. 2010. "Perceived Stress Scale: Reliability and validity study in Brazil.” Journal of Health Psychology 15(1): 107-14. doi:10.1177/1359105309346343

Reise, S.P., T.M. Moore, and M.G. Haviland. 2010. "Bifactor models and rotations: Exploring the extent to which multidimensional data yield univocal scale scores." Journal of Personality Assessment 92(6): 544-559. doi:10.1080/00223891.2010.496477

Reise, S.P., J. Morizot, and R.D. Hays. 2007. "The role of the bifactor model in resolving dimensionality issues in health outcomes measures." Quality of Life Research 16(1): 19-31. doi:10.1007/s11136-007-9183-7

Roberti, J.W., L.N. Harrington, and E. Storch. 2006. "Further psychometric support for the 10-item version of the Perceived Stress Scale.” Journal of College Counseling 9 (2): 135-47. doi:10.1002/j.2161-1882.2006.tb00100.x

Roediger, H.L., A.L. Putnam, and M.A. Smith. 2011. "Ten Benefits of Testing and Their Applications to Educational Practice.” In The Psychology of Learning and Motivation, ed. J. Mestre and B. Ross., 1-37. Amsterdam: Academic Press. 
Schiffrin, H.H., and S.K. Nelson. 2010. "Stressed and happy? Investigating the relationship between happiness and perceived stress." Journal of Happiness Studies 11(1): 33-39. doi:10.1007/s10902-008-9104-7

Shevlin, M.E., J.N.V. Miles, M.N.O. Davies, and S. Walker. 2000. "Coefficient alpha: A useful indicator of reliability?" Personality and Individual Differences 28(2): 229238. doi:10.1016/S0191-8869(99)00093-8

Smith, K.J., D.L. Rosenberg, and G.T. Haight. 2014. “An assessment of the psychometric properties of the Perceived Stress Scale-10 (PSS10) with business and accounting students.” Accounting Perspectives 13(1): 29-59. doi:10.1111/1911-3838.12023

Stallman, H.M. 2010. "Psychological distress in university students: A comparison with general population data." Australian Psychologist 45(4): 249-257. doi:10.1080/00050067.2010.482109

Steiger, J.H. 1990. "Structural model evaluation and modification: An interval estimation approach.” Multivariate Behavioural Research 25(2): 173-180. doi:10.1207/s15327906mbr2502_4

Struthers, C.W., R.P. Perry, and V.H. Menec. 2000. "An examination of the relationship among academic stress, coping, motivation, and performance in college." Research in higher education 41(5): 581-592. doi:10.1023/A:1007094931292

Taylor, J.M. 2015. "Psychometric analysis of the Ten-Item Perceived Stress Scale." Psychological assessment 27(1): 90-101. doi:10.1037/a0038100

ten Klooster, P.M., M.M. Veehof, E. Taal, P.L. van Riel, P. L. and M.A. van de Laar. 2008. "Confirmatory factor analysis of the Arthritis Impact Measurement Scales 2 short form in patients with rheumatoid arthritis." Arthritis Care \& Research 59(5): 692698. doi:10.1002/art.23569 
Tucker, L.R., and C. Lewis. 1973. "The reliability coefficient for maximum likelihood factor analysis." Psychometrika 38: 1-10. doi:10.1007/BF02291170

Turner, J., D. Bartlett, M. Andiappan, and L. Cabot. 2015. “Students' perceived stress and perception of barriers to effective study: Impact on academic performance in examinations.” British dental journal 219(9): 453-458. doi:10.1038/sj.bdj.2015.850

Vaez, M., M. Kristenson, and L. Laflamme. 2004. "Perceived quality of life and self-rated health among first-year university students. A comparison with their same age working peers." Social Indicators Research 68(2): 221-234. doi:10.1023/B:SOCI.0000025594.76886.56

Warttig, S.L., M.J. Forshaw, J. South, and A.K. White. 2013. "New, normative, Englishsample data for the short form perceived stress scale (PSS-4)." Journal of health psychology 18(12): 1617-1628. doi:10.1177/1359105313508346

Watson, D., L.A. Clark, and A. Tellegen. 1988. "Development and validation of brief measures of positive and negative affect: The PANAS Scales.” Journal of Personality and Social Psychology 54(6): 1063-1070. doi:10.1037/0022-3514.54.6.1063

Wongpakaran, N., and T. Wongpakaran. 2010. "The Thai version of the PSS-10: An investigation of its psychometric properties.” BioPsychoSocial Medicine 4(6): 1-6. doi:10.1186/1751-0759-4-6

Wu, S.M., and D. Amtmann. 2013. "Psychometric evaluation of the perceived stress scale in multiple sclerosis.” ISRN Rehabilitation 1-9. doi:10.1155/2013/608356

Zhang, B., X. Yan, F. Zhao, and F. Yuan. 2015. "The relationship between perceived stress and adolescent depression: The roles of social support and gender." Social Indicators Research 123(2): 501-518. doi:10.1007/s11205-014-0739-y 
Perceived Stress Scale - 10 Item (Cohen and Williamson 1988)

Instructions: The questions in this scale ask you about your feelings and thoughts during the last month.

1. In the last month, how often have you been upset because of something that happened unexpectedly?

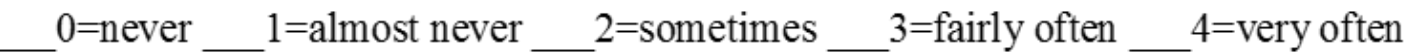

2. In the last month, how often have you felt that you were unable to control the important things in your life?

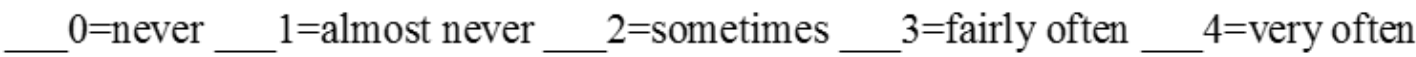

3. In the last month, how often have you felt nervous and "stressed"?

_ $0=$ never __ $1=$ =almost never __ $2=$ sometimes __ 3 =fairly often __ $4=$ very often

4. In the last month, how often have you felt confident about your ability to handle your personal problems?

$0=$ never __ $1=$ =almost never __ $2=$ sometimes __ 3=fairly often __ $4=$ =very often

5. In the last month, how often have you felt that things were going your way?

$0=$ never __ $1=$ =almost never __ $2=$ sometimes __ 3 =fairly often __ $4=$ =very often

6. In the last month, how often have you found that you could not cope with all the things that you had to do?

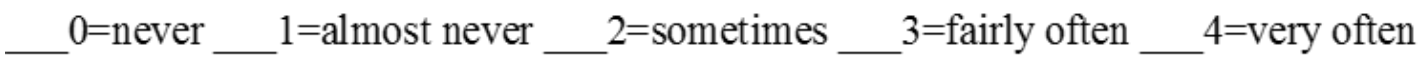

7. In the last month, how often have you been able to control irritations in your life?

$0=$ never $\_1=$ almost never __ $2=$ sometimes _ _ $3=$ fairly often $\_4=$ very often

8. In the last month, how often have you felt that you were on top of things?

$0=$ never __ $1=$ almost never __ $2=$ sometimes __ 3 =fairly often __ $4=$ =very often

9. In the last month, how often have you been angered because of things that were outside of your control?

${ }^{0} 0=$ never __ $1=$ almost never __ $2=$ sometimes __ 3 =fairly often __ $4=$ very often 10. In the last month, how often have you felt difficulties were piling up so high that you could not overcome them?

$0=$ never __ $1=$ =almost never __ $2=$ sometimes __ 3 =fairly often __ $4=$ =very often 
Table 1. Summary statistics for all study variables

\begin{tabular}{|c|c|c|c|c|c|c|c|c|c|c|c|c|c|c|c|}
\hline \multirow[b]{2}{*}{ Variable } & \multicolumn{5}{|c|}{ Total sample } & \multicolumn{5}{|c|}{ Males } & \multicolumn{5}{|c|}{ Females } \\
\hline & $\mathrm{M}$ & SD & Range & Kurtosis & Skewness & $\mathrm{M}$ & SD & Range & Kurtosis & Skewness & $\mathrm{M}$ & SD & Range & Kurtosis & Skewness \\
\hline PS Total & 19.79 & 6.37 & 38.0 & .05 & .04 & 19.25 & 6.76 & 37.0 & -.12 & .04 & 20.19 & 6.03 & 36.0 & .09 & .17 \\
\hline Distress & 12.47 & 4.44 & 24.0 & -.04 & .17 & 12.07 & 4.68 & 23.0 & -.19 & .09 & 12.77 & 4.23 & 22.0 & .04 & .30 \\
\hline Coping & 7.31 & 2.77 & 15.0 & -.18 & .12 & 7.18 & 2.94 & 15.0 & -.33 & .21 & 7.41 & 2.64 & 15.0 & -.02 & .05 \\
\hline Positive Affect & 33.14 & 7.31 & 38.0 & -.14 & -.19 & 32.71 & 7.69 & 38.0 & -.10 & -.14 & 33.46 & 7.01 & 38.0 & -.22 & -.23 \\
\hline Negative Affect & 24.68 & 7.84 & 38.0 & -.59 & .28 & 23.85 & 8.15 & 37.0 & -.68 & .32 & 25.30 & 7.54 & 37.0 & -.50 & .29 \\
\hline ICSRLE Total & 75.03 & 19.09 & 90.0 & .16 & .88 & 72.56 & 17.43 & 88.0 & .44 & .93 & 78.33 & 20.70 & 88.0 & -.28 & .74 \\
\hline $\begin{array}{l}\text { Developmental } \\
\text { Challenge }\end{array}$ & 20.85 & 5.10 & 29.0 & -.42 & .33 & 20.57 & 5.09 & 29.0 & -.26 & .45 & 21.23 & 5.10 & 25.0 & -.54 & .16 \\
\hline Time Pressure & 15.06 & 4.27 & 19.0 & -.03 & .64 & 14.90 & 4.05 & 19.0 & -.10 & .57 & 15.28 & 4.54 & 18.0 & -.07 & .67 \\
\hline $\begin{array}{l}\text { Academic } \\
\text { Alienation }\end{array}$ & 6.48 & 3.54 & 15.0 & -.05 & 1.03 & 6.14 & 3.27 & 14.0 & .50 & 1.19 & 6.94 & 3.84 & 15.0 & -.60 & .82 \\
\hline $\begin{array}{l}\text { Romantic } \\
\text { Problems }\end{array}$ & 6.77 & 3.11 & 14.0 & .15 & .86 & 6.35 & 2.81 & 14.0 & .90 & 1.01 & 7.33 & 3.39 & 14.0 & -.52 & .63 \\
\hline $\begin{array}{l}\text { Assorted } \\
\text { Annoyances }\end{array}$ & 8.78 & 2.99 & 12.0 & .16 & .89 & 8.59 & 2.73 & 12.0 & .24 & .79 & 9.04 & 3.29 & 12.0 & -.19 & .89 \\
\hline $\begin{array}{l}\text { General Social } \\
\text { Mistreatment }\end{array}$ & 10.91 & 4.35 & 18.0 & 1.16 & 1.23 & 10.27 & 3.78 & 18.0 & 1.49 & 1.27 & 11.79 & 4.90 & 18.0 & .40 & 1.04 \\
\hline $\begin{array}{l}\text { Friendship } \\
\text { Problems }\end{array}$ & 6.14 & 3.33 & 15.0 & 1.13 & 1.37 & 5.73 & 3.04 & 15.0 & 1.57 & 1.66 & 6.69 & 3.62 & 15.0 & .03 & 1.06 \\
\hline
\end{tabular}

Note PS Total $=$ Perceived Stress Total; ICSRLE Total $=$ Inventory of College Students' Recent Life Experiences Total 
Table 2. Fit indices for alternative PSS-10 measurement models

\begin{tabular}{llllllll}
\hline & $\chi 2$ & $d f$ & CFI & TLI & SRMR & RMSEA (90\% CI) & AIC \\
\hline One-factor & $299.97 * *$ & 35 & .85 & .81 & .07 & $.12(.11-.13)$ & 339.97 \\
One-factor with & $104.71 * *$ & 30 & .95 & .93 & .05 & $.07(.06-.08)$ & 174.71 \\
correlated errors & & & & & & & \\
Correlated two- & $108.21^{* *}$ & 34 & .96 & .95 & .03 & $.06(.05-.08)$ & 170.21 \\
factor & & & & & & & \\
Two-factor with & $65.95 *$ & 33 & .98 & .97 & .03 & $.04(.03-.06)$ & 129.95 \\
correlated errors & & & & & & .03 & \\
Correlated three- & $200.50 * *$ & 32 & .91 & .87 & .06 & $.10(.09-.11)$ & 266.50 \\
factor & & & & & & & \\
Bifactor & $45.72 *$ & 25 & .99 & .98 & .02 & $.04(.02-.06)$ & 125.72 \\
\hline
\end{tabular}

Note. $\chi^{2}=$ chi-square goodness-of-fit statistic; $d f=$ degrees of freedom; $\mathrm{CFI}=\mathrm{Comparative}$

Fit Index; TLI = Tucker-Lewis Index; SRMR = Standardized Root-Mean-Square Residual;

RMSEA = Root-Mean-Square Error of Approximation; AIC = Akaike Information Criterion;

$* \chi^{2}$ significant at $p<.05 ; * * \chi^{2}$ significant at $p<.001$ 
Table 3. Correlations of positive affect, negative affect, ICSRLE total, and ICSRLE subscales with PSS-10 total and subscales

Two-factor PSS-10 version

\begin{tabular}{llll}
\hline Variable & PS Total & Distress & Coping \\
\hline Positive Affect & $-.39^{* *}$ & $-.30^{* *}$ & $-.40^{* *}$ \\
Negative Affect & $.65^{* *}$ & $.64^{* *}$ & $.48^{* *}$ \\
ICSRLE Total & $.09^{*}$ & .08 & .07 \\
Developmental Challenge & $.10^{*}$ & $.10^{*}$ & .08 \\
Time Pressure & $.12^{* *}$ & $.12^{* *}$ & .08 \\
Academic Alienation & -.07 & -.05 & -.08 \\
Romantic Problems & .01 & .01 & .02 \\
Assorted Annoyances & .08 & .07 & .07 \\
General Social & .07 & .07 & .06 \\
Mistreatment & & & .07 \\
Friendship Problems & .08 & & \\
\hline
\end{tabular}

Note. PS Total $=$ Perceived Stress Total; ICSRLE Total $=$ Inventory of College Students'

Recent Life Experiences Total; *Correlation significant at $p<.05 ; * *$ Correlation significant at $p<.001$ 


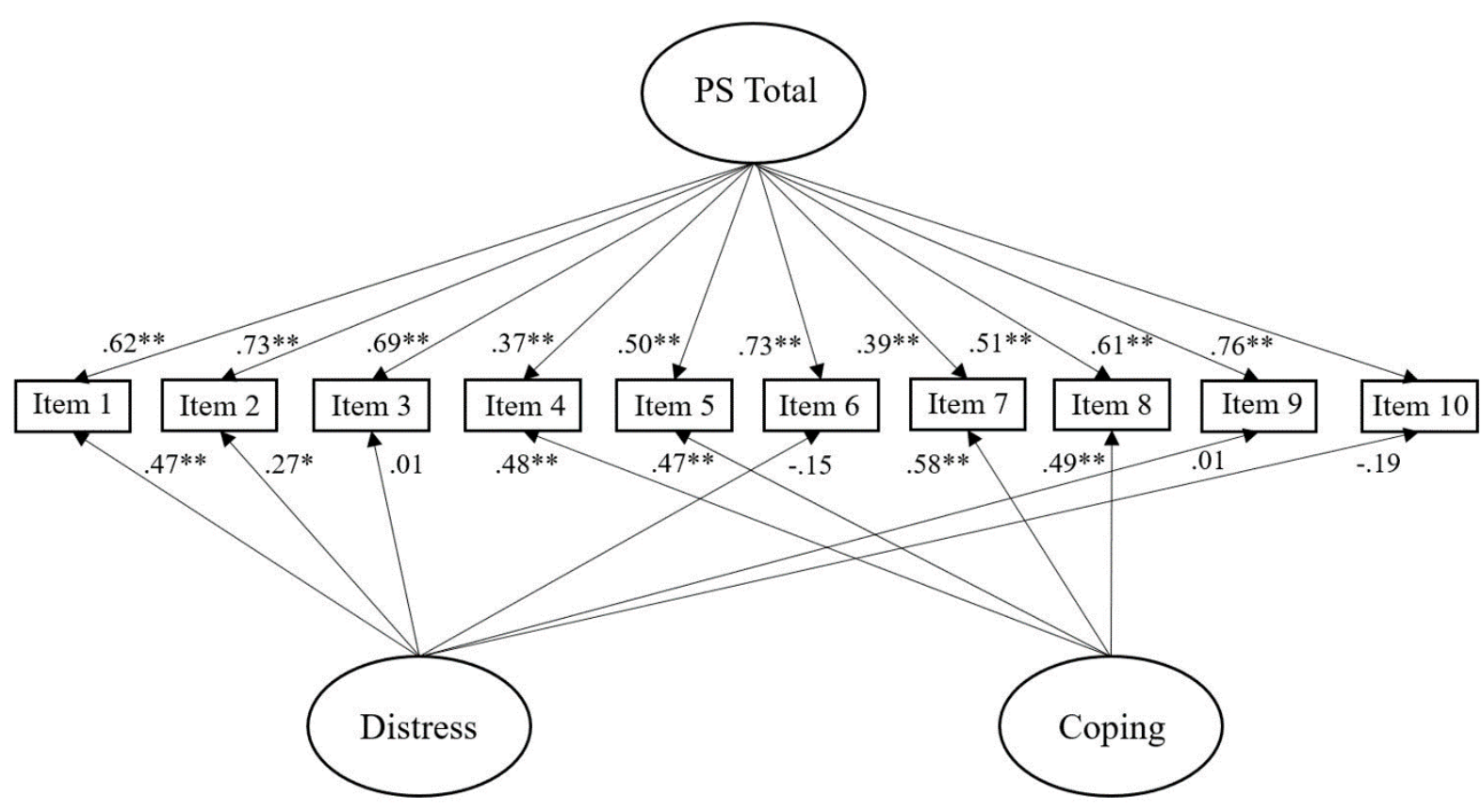

Figure 1. Bifactor solution of PSS-10. Latent variables are represented by ellipses; measured variables are represented by rectangles. Error of measurement is not shown, but was specified for each variable in the model. *Factor loading significant at $p<.05$; **Factor loading significant at $p<.001$. 